\title{
RESEARCH IN GLOBAL DYNAMICS OF DUFFING-TYPE OSCILLATOR BY METHOD OF COMPLETE BIFURCATION ANALYSIS
}

\author{
Raisa Smirnova, Jurij Ivanov, Vladimir Nikishin \\ Riga Technical University, Latvia \\ raja@df.rtu.lv,ijm@df.rtu.lv, nikis@df.rtu.lv
}

\begin{abstract}
Numerous researches of the Duffing-type oscillator have revealed a big variety of dynamics of this nonlinear system. Nonlinear effects, which result from changes of initial conditions and parameters, can be manifested in various, sometimes seemingly unexpected, ways. The task of studying nonlinear effects has important practical value for engineering dynamical systems working by the Duffing-type oscillator. For the research of nonlinear effects we usually use the method of harmonic balance, analog simulation or the method of direct scanning. Such techniques are a little limited regarding the ability to predict all dynamical characteristics, including bifurcations that lead to occurrence of unstable, subharmonic and chaotic solutions. The given work is devoted to the study of complicated dynamics of the Duffing-type oscillator and the reasons of its occurrence at the change of the system parameters on the basis of the system approach by the method of complete bifurcation analysis. The results are received by direct numerical simulation with the use of software NLO and SPRING. Bifurcation diagrams at the research of dynamics of the Duffing-type oscillator are constructed by means of the parameter continuation approach of steady and unstable periodic solution. The example of the Duffing-type oscillator illustrates high efficiency of the method of complete bifurcation analysis for providing an explanation of the reasons of the birth of regular and chaotic solutions, their coexistence and influence on transient processes. At the systematic approach to certain parameters, at which the complete bifurcation analysis specifies the preconditions of the birth of unexpected nonlinear effects, in the investigated system an additional analysis of various periodic and chaotic attractors, forecasting of rare attractors and rare phenomena, studying of scenarios of their birth is carried out.
\end{abstract}

Keywords: nonlinear effect, dynamical system, bifurcation diagram, rare attractor, chaos.

\section{Introduction}

An important task for all nonlinear dynamical systems is the study of the nonlinear effects that allow predicting and explaining complicated behaviour of such systems. Modern researches of nonlinear phenomena in the nature and engineering are related to the study of typical structures in the behaviour of nonlinear dynamical systems. This work is devoted to the research of regular and chaotic dynamics of the Duffing-type oscillator and systematic revealing of nonlinear effects. The work illustrates the advantage of the method of complete bifurcation groups for the research of nonlinear effects in comparison to the applied methods: harmonic balance and direct scanning.

\section{Dynamical model and bifurcation analysis for regular and chaotic dynamics research}

The aim of the given work is to research in the dynamics of the Duffing-type oscillator (1), study regular and chaotic solutions, nonlinear effects [1-3]. The authors apply up-to-date numerical and analytical methods of the analysis of nonlinear dynamical systems. Firstly, these are the points mapping method and the parameter continuation method. It is offered to apply a systematic approach to the research of periodic and chaotic forced oscillations in nonlinear systems: search of periodic regimes, contour mapping, constructing of regime attraction domains, construction of bifurcation diagrams.

In this paper, periodic and non-periodic steady or transition oscillations of the influence of the frequency of external harmonic forcing are observed in computer-simulated solutions of the secondorder nonlinear ordinary differential equation of the Duffing-type oscillator (1):

$$
\ddot{x}+b \dot{x}+a_{1} x+a_{3} x^{3}=h \cos (\omega t),
$$

where $x$-displacement;

$a_{1}$ and $a_{3}$ - coefficients of the Duffing-type restoring force;

$b$ - dissipation coefficient of the linear dissipative force;

$F(t)=h \cos (\omega t)-$ harmonic driving force with the period $T=2 \pi / \omega$;

$h$ - amplitude;

$\omega=w-$ frequency. 
Let us show the application of the method of harmonic balance [4] for the research of the Duffingtype oscillator. At calculation by the method of harmonic balance on the first harmonics the solution of equation (1) is set in the form of (2). Parameters A and B are unknown values. Solution (2) is placed in equation (1) and the amplitudes are equated at the sine and cosine of the first harmonics. After the transformations we receive a system of two nonlinear algebraic equations (3). The system of equations (3) is solved numerically at zero initial approach in software MATLAB.

System (3) is reduced to one cubic equation (4) for the amplitude of the sinus components of the solution A. We find the root of cubic equation (4) and determine the amplitude of the cosine component of the solution B based on expression (5). Cubic equation (4) is recorded in the canonical form (6). For the canonical form (6) the discriminant of the equation is (7). At a positive discriminant (7) cubic equation (4) has one real root; at a negative discriminant the cubic equation will have three real roots (Fig.1.). The system described by equation (1) possesses one or three possible regimes according to the quantity of real roots of cubic equation (4). In case of coexistence of three regimes of the basic group there is, so-called in the electrical engineering, the trigger or hysteresis effect when there is a discontinuous change of the regime at the change (increase and reduction) of the parameters (see Fig. 1).

$$
\begin{gathered}
x=A \sin (\omega t)+B \cos (\omega t) . \\
\left\{\begin{array}{l}
\left(a_{1}-\omega^{2}\right) A-b \omega B+\frac{3}{4} a_{3} A\left(A^{2}+B^{2}\right)=0 \\
b \omega A+\left(a_{1}-\omega^{2}\right) B+\frac{3}{4} a_{3} B\left(A^{2}+B^{2}\right)=h
\end{array}\right. \\
\frac{9}{16} \cdot \frac{\left(a_{3} h\right)^{2}}{(b \omega)^{3}} \cdot A^{3}+\frac{3}{2} \cdot \frac{a_{3} h\left(a_{1}-\omega^{2}\right)}{(b \omega)^{2}} \cdot A^{2}+\left(b \omega+\frac{\left(a_{1}-\omega^{2}\right)^{2}}{b \omega}\right) \cdot A-h=0 . \\
B=\frac{\left(a_{1}-\omega^{2}\right)}{b \omega} \cdot A+\frac{3}{4} \cdot \frac{a_{3} \cdot h}{(b \omega)^{2}} \cdot A^{2} . \\
r=\frac{8}{3} \cdot \frac{\omega b\left(a_{1}-\omega^{2}\right)}{a_{3} h}, \\
s=\frac{16}{9} \cdot \frac{(b \omega)^{2}}{\left(a_{3} h\right)^{2}} \cdot\left(b^{2} \omega^{2}+\left(a_{1}-\omega^{2}\right)^{2}\right), \\
z=-\frac{16}{9} \cdot \frac{(\omega b)^{3}}{h \cdot a_{3}^{2}} . \\
D=\frac{p^{3}}{27}+\frac{q^{2}}{4}, \frac{p}{p=} \cdot \frac{1}{3} \cdot r^{2}, q=\frac{2}{27} \cdot r^{3}-\frac{1}{3} \cdot r \cdot s+z .
\end{gathered}
$$

When calculating with the help of the harmonic balance method on the first and third harmonics the solution of equation (1) is set in the form of (8). Parameters $A, B, F, G$ are unknown values. Solution (8) is placed in equation (1) and the amplitudes are equated at the sine and cosine of the first and third harmonics. After the transformations we receive a system of four nonlinear algebraic equations (9). The system of equations (9) is solved numerically at zero initial approach in software MATLAB.

$$
x=A \sin (\omega t)+B \cos (\omega t)+F \sin (3 \omega t)+G \cos (3 \omega t)
$$




$$
\left\{\begin{array}{l}
4 \cdot \frac{\left(a_{1}-\omega^{2}\right)}{3 \cdot a_{3}} \cdot A-b \omega \cdot B+A^{3}+A \cdot B^{2}+2 \cdot A \cdot F^{2}-A^{2} \cdot F+B^{2} \cdot F+2 \cdot A \cdot G^{2}-4 \cdot A B G=0 \\
\frac{4 \cdot b \omega}{3 \cdot a_{3}} \cdot A+4 \cdot \frac{\left(a_{1}-\omega^{2}\right)}{3 \cdot a_{3}} \cdot B+B^{3}+A^{2} B+2 \cdot B F^{2}-A^{2} G+2 B G^{2}+B^{2} \cdot G+2 A B F-2 A F G=\frac{4 \cdot h}{3 \cdot a_{3}} \\
-\frac{1}{3} A^{3}+A B^{2}+\frac{4\left(a_{1}-9 \omega^{2}\right)}{3 \cdot a_{3}} \cdot F-\frac{4 b \omega}{a_{3}} \cdot G+F^{3}+2 A^{2} F+2 B^{2} F+F G^{2}-2 A B G=0 \\
\frac{1}{3} B^{3}-A^{2} B+\frac{4 b \omega}{a_{3}} \cdot F+\frac{4\left(a_{1}-9 \omega^{2}\right)}{3 \cdot a_{3}} \cdot G+G^{3}+2 A^{2} G+2 B^{2} G+F^{2} G-4 A B F+2 A F G=0
\end{array}\right.
$$
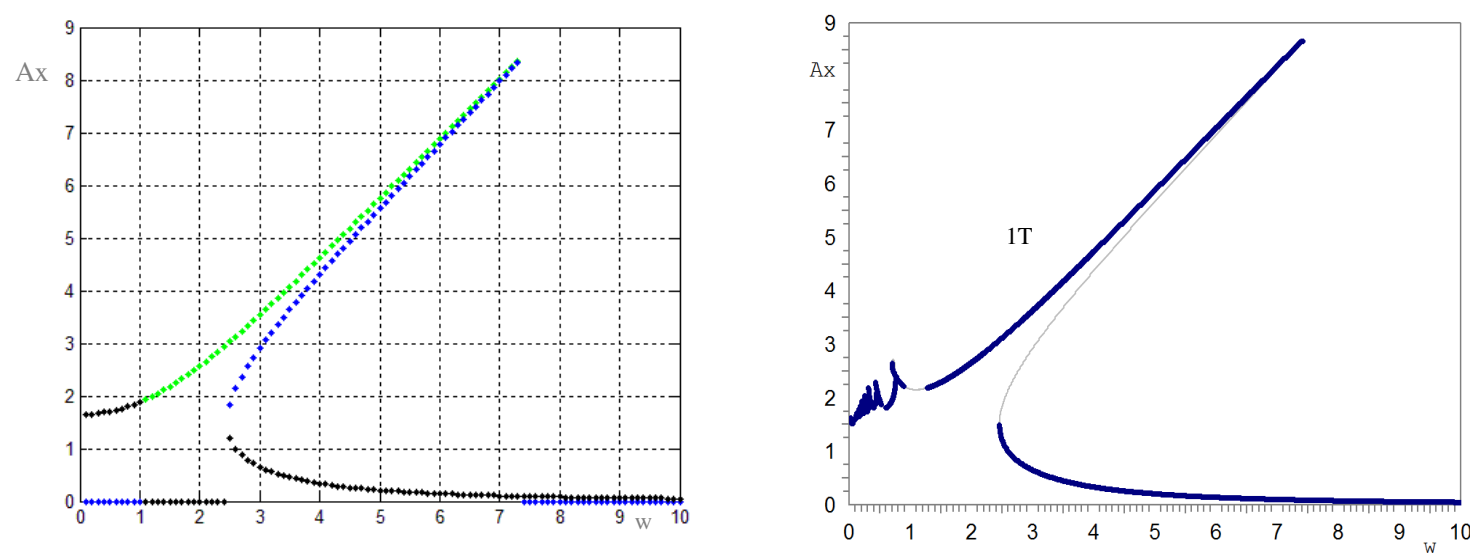

Fig. 1. Bifurcation diagrams of the Duffing-type oscillator of basic periodic regimes at the change of the frequency $\omega$ of external harmonic forcing applying the harmonic balance method on the first harmonics (left fig.) and applying the method of complete bifurcation analysis of the parameter continuation approach of stable (dark gray) and unstable (light gray) periodic solutions with the basic bifurcation group 1T (right fig.). System parameters:

$$
a_{1}=1, a_{3}=1, b=0.08, h=5, \omega=w=v a r
$$

The method of harmonic balance (HBM) is the approached method of definition of periodic regimes in nonlinear oscillatory systems, based on the theory that at presence of nonlinearity the established oscillations are frequently close to the harmonic. The HBM is also suitable for the study of oscillations of autonomous systems. The HBM in many cases can give successful results in terms of quality and often in quantity not only at small nonlinearities, but also for essentially nonlinear systems. High efficiency HBM in the research of the basic regime can be explained by filtering properties of many nonlinear systems, which allows approximating the solution by the sum of harmonics. For the research of sub-harmonic and chaotic solutions the applicability of the method is strongly limited by the complexity of transformations (see Fig. 2 and Fig. 3).

For the display of regular and chaotic stationary regimes (attractors) in bifurcation diagrams the scanning method (a method of direct scanning) is usually used [5]. This method allows defining quantitative values of phase coordinates of attractors that correspond to the results of the integration after the completion of the transition process at the movement on the parameter of one or several initial conditions belonging to the area of attraction of the stationary regime (see Fig. 4). To receive a complete bifurcation image we need to carry out a whole set of additional researches at fixed values of parameters in order to define the quantity of attractors, which carries occasional rather than systematic character.

The idea of the RTU professor M. Zakrzhevsky of continuation of steady and unstable solutions of one bifurcation group before the construction of a complete bifurcation image allows carrying out a complete bifurcation analysis and systematically find and explain nonlinear effects. Under the supervision of M. Zakrzhevsky the Spring and NLO software have been developed for the research of dynamical systems [6-8]. 
The method of complete bifurcation groups and the complex of algorithms developed on its basis and the software allow finding qualitatively new previously unfamiliar regular and chaotic regimes, rare attractors (RA) and rare phenomena, new bifurcation groups for typical and widely applied classical nonlinear dynamical models and studying the interaction of various bifurcation groups [8-9]. The completeness of a bifurcation group is defined, if we find for it all steady and unstable regimes and also connected with this bifurcation group in space of parameters of dissipative nonlinear dynamical systems. At variation of parameters in nonlinear oscillatory systems qualitative changes in behaviour of the system take place, which leads to the loss of stability of the periodic solution, the cascade bifurcations of period doubling, to the formation of areas with infinite number of unstable regimes UPI (Unstable Periodic Infinitium) and to the birth of chaotic attractors [10]. In nonlinear dynamical systems the birth of chaotic oscillations is connected with the basic bifurcation group 1T, subharmonic bifurcation groups $\mathrm{nT}$ and their coexistence [11].
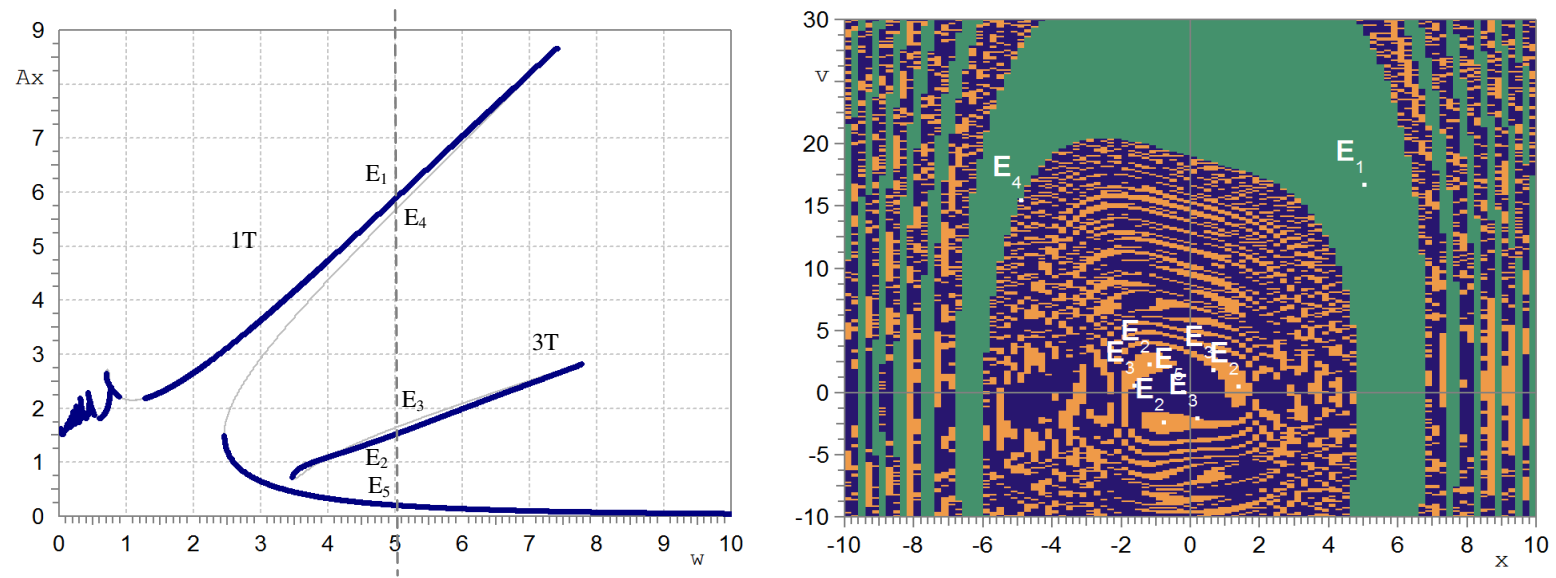

Fig. 2. Bifurcation diagram of the Duffing-type oscillator applying the method of complete bifurcation analysis of the parameter $\omega$ continuation approach of steady and unstable periodic solution with the basic bifurcation group 1T and subharmonic bifurcation group $3 T$ (left fig.).

System parameters: $a_{1}=1, a_{3}=1, b=0.08, h=5, \omega=\mathrm{w}=$ var. Nonlinear multistability phenomenon of the coexistence of attractors: at $w=5$ the right figure displays all the domains of attraction for stable periodic regimes $E_{1}, E_{2}, E_{5}$ and coordinates for unstable periodic regimes $E_{3}, E_{4}$ (see Fig. 3)
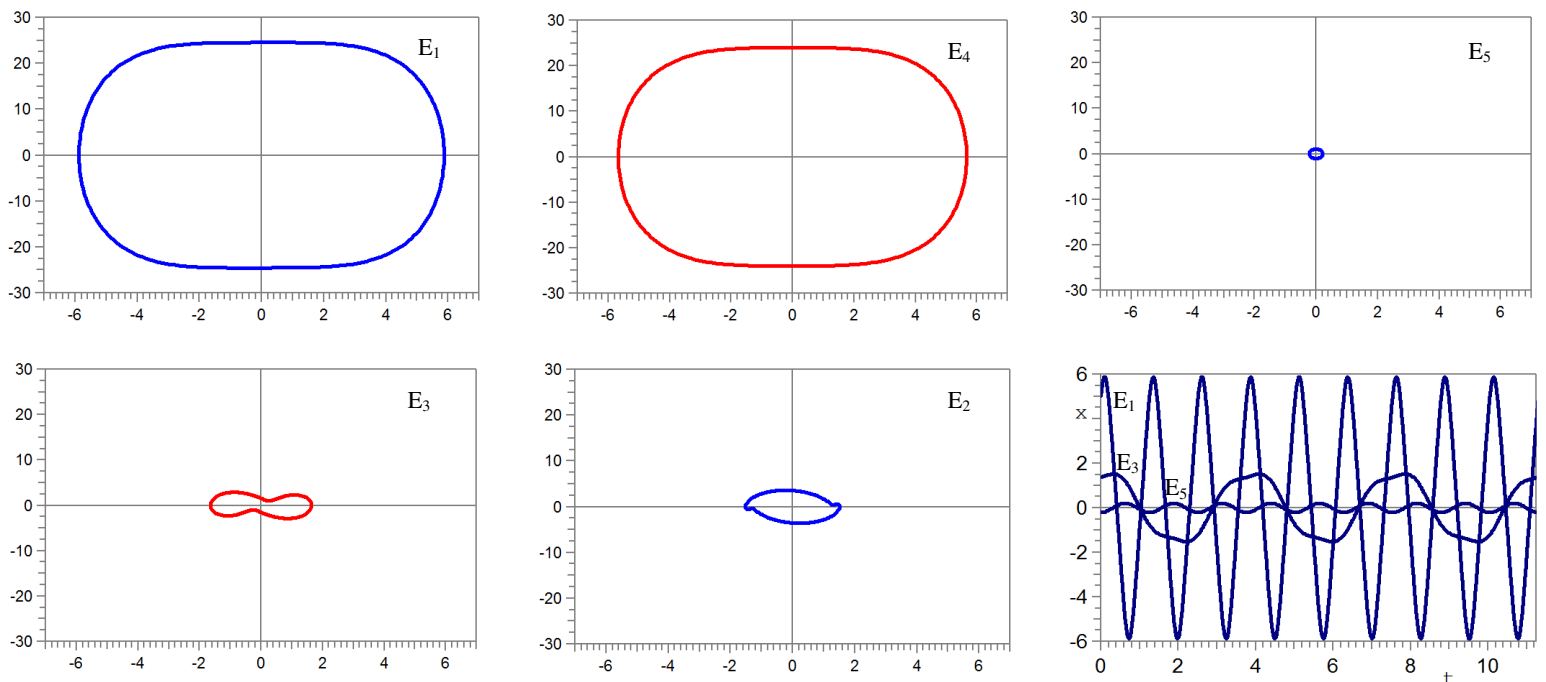

Fig. 3. Phase portraits and phase trajectories of stable periodic regimes (attractors $E_{1}, E_{2}, E_{5}$ ) and phase portraits of unstable periodic regimes $E_{3}, E_{4}$ from the bifurcation groups $1 T$ and $3 T$ (see Fig. 2). Initial conditions: $E_{1}(x=5.0224, v=16.752981) ; E_{2}(x=1.374577, v=0.514462)$; $E_{3}(x=-1.624419, v=0.59085) ; E_{4}(x=-4.914975, v=15.491486) ; E_{5}(x=-0.208568, v=0.017412)$. The nonlinear driven Duffing-type oscillator parameters: $a_{1}=1, a_{3}=1, b=0.08, h=5, w=5$ 
For display of the chaotic transient process, regular and chaotic attractors in bifurcation diagrams the scanning method is used to determine the phase space of the quantitative value.
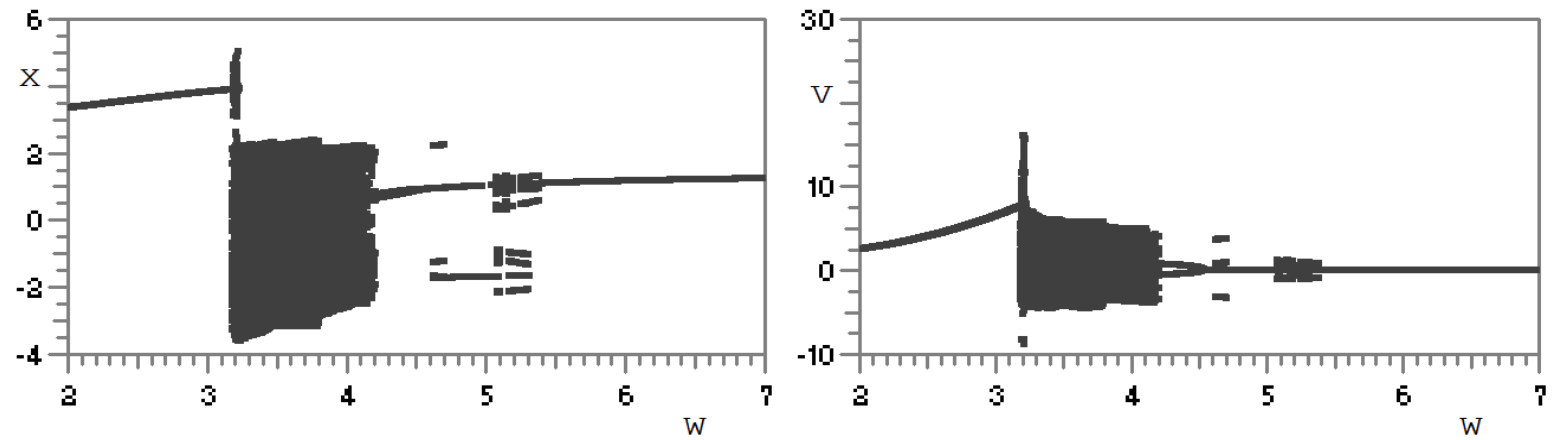

Fig. 4. Bifurcation diagram (dependence of stationary regular periodic and chaotic solutions displacement $x$ and velocity $v$ ) of the Duffing-type oscillator applying the scanning method

(a method of direct scanning) on the excitation force frequency $w$. System parameters:

$$
a_{1}=-2, a_{3}=1, b=0.2, h=7, \omega=w=v a r
$$
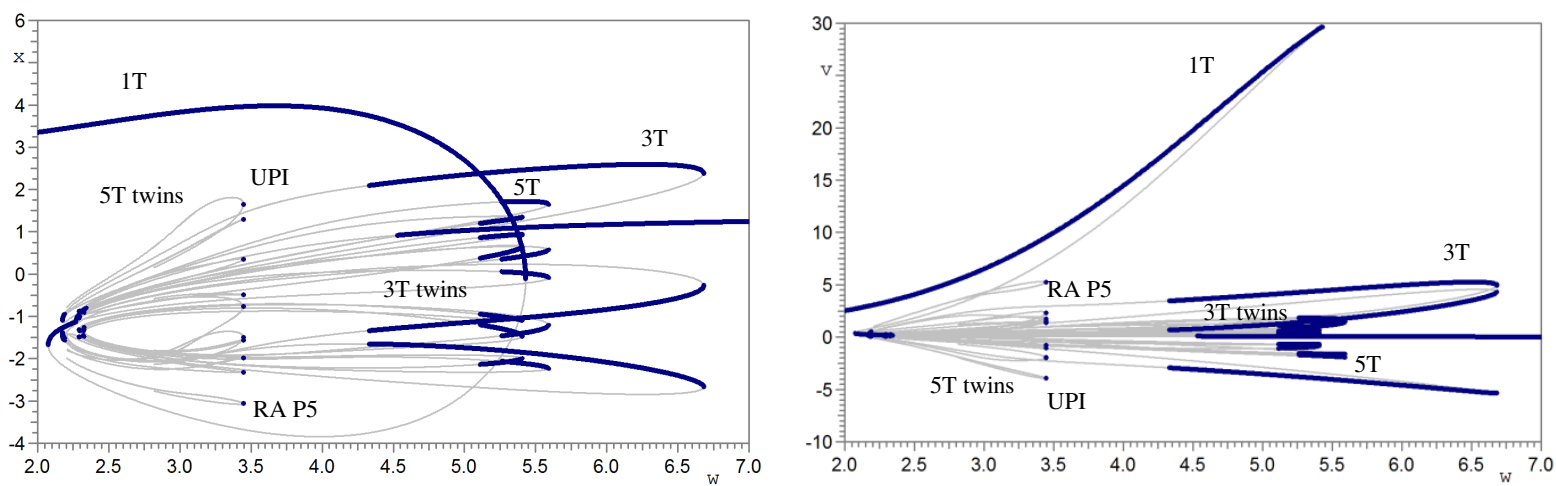

Fig. 5. Complete bifurcation diagram of stable (dark gray) and unstable (light gray) periodic solutions (dependence displacement $x$ and velocity $v$ ) and chaotic motions or attractors (UPI regions) of 7 bifurcation groups (1T, three $3 T$ and three $5 T$ ) of the driven Duffing-type oscillator applying the method of complete bifurcation analysis of the parameter $w$ (frequency of external harmonic forcing) continuation. New nonlinear phenomena have been discovered: rare attractors (RA) (see Fig. 6). System parameters: $a_{1}=-2, a_{3}=1, b=0.2, h=7, \omega=w=v a r$
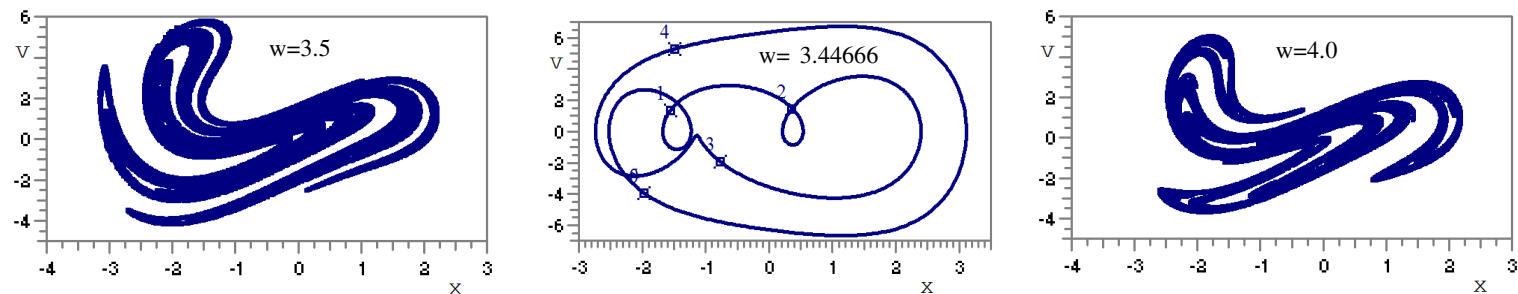

Fig. 6. Chaotic attractors $(w=3.5$ and $w=4.0)$ from initial conditions $(x=-2, v=0)$ during 50000 periods on the Poincaré plane and the phase portrait of subharmonic solution - attractor $P_{5}$ of

the Duffing-type oscillator. At the value of the parameter $w=3.44666$, we can observe a transient chaos (chaotic oscillations) during 29740 periods from initial conditions $(x=-2, v=0)$.

Then, periodic attractor $P_{5}$ (rare attractor RA) is formed in the system. It is displayed in the middle image. The nonlinear driven Duffing-type oscillator parameters: $a_{1}=-2, a_{3}=1, b=0.2$,

$$
h=7, w=v a r
$$

Efficient algorithms of software Spring allow conducing researches of regular and chaotic solutions of all types according to the method of complete bifurcation groups. The examples in Fig. 5 of the bifurcation analysis for the Duffing-type oscillator by means of software Spring illustrate the advantage and systematicity of the research of dynamical systems applying the method of complete bifurcation groups. 


\section{Results and discussion}

This work shows the advantage of the method of complete bifurcation groups in comparison to usually applied methods of harmonic balance and direct scanning, which do not allow complete predicting of many nonlinear effects, including bifurcations that lead to the occurrence of unstable, sub-harmonic and chaotic solutions. With the example of the Duffing-type oscillator the authors have considered and illustrated new possibilities in the research of the birth and existence of nonlinear effects, for example, such as multistability, chaotic oscillations and rare attractors. These new possibilities have appeared owing to the method of complete bifurcation groups, which is the basis of the search of all basic periodic solutions and their continuation in the investigated parameter, studying the topology of their bifurcations. Thus, bifurcation diagrams display all bifurcation groups, which allow studying the birth of nonlinear effects, analyzing interaction of bifurcation groups at the change of parameters. A typical element of a bifurcation group with a chaotic attractor is the areas in space of parameters with infinite number of unstable periodic regimes resulting from the cascade of bifurcations of period doubling. The systematic approach to carrying out bifurcation analysis on the basis of the method of complete bifurcation groups allows explaining the origin of rare attractors nonlinear effects that are hard to be diagnosed and which exist in a small interval of a changeable parameter.

\section{Conclusions}

1. The example of the Duffing-type oscillator has been used to show the advantage of carrying out a bifurcation analysis with the application of the method of complete bifurcation groups.

2. The analysis of coexistence of various bifurcation groups has enabled the systematic study of mechanisms of the birth of nonlinear effects in the investigated dynamical system.

3. It has been shown that the method of complete bifurcation groups allows defining a way to chaotic oscillations and rare attractors.

4. The results of the research of attractors, the birth of which is closely connected with unstable solutions, can be used to forecast and avoid catastrophic situations in electric mechanical and other nonlinear dynamical systems.

\section{References}

1. Duffing G., Erzwungene Schwingungen bei veranderlicher Eigenfrequenz und ihre technische Bedeutung, F. Vieweg, Braunschweig, 41/42, 1918.

2. Kovacic I., Brennan M.J. The Duffing Equation: Nonlinear Oscillators and their Behaviour. John Wiley and Sons, Chichester, 2011, 390 p.

3. Ueda Y. The Road to Chaos - II, Aerial Press Inc. - Santa Cruz, 2001.

4. Vibrations in Engineering: Reference Book. In 6 volumes. M.: Machine Engineering, V.2. Oscillations of Nonlinear Mechanical Systems/Edited by I.I. Blehman. 1979, p.351 (in Russian).

5. Lakshmanan M., Rajasekar S. Nonlinear Dynamics. Integrability, Chaos and Patterns. SpringerVerlag, Berlin, Heidelberg, 2003.

6. Zakrzhevsky M., Ivanov Yu., Frolov V. NLO: Universal Software for Global Analysis of Nonlinear Dynamics and Chaos. In Proceeding of the 2nd ENOC, Prague 1996. v.2. pp.261-264.

7. Zakrzhevsky M. New concepts of nonlinear dynamics: complete bifurcation groups, protuberances, unstable periodic infinitiums and rare attractors. Journal of Vibroengineering, Volume 10, Issue 4, 2008, pp. 421-441.

8. Schukin I., Zakrzhevsky M., Ivanov Yu., Kugelevich V., Malgin V., Frolov V. Application of software SPRING and method of complete bifurcation groups for the bifurcation analysis of nonlinear dynamical system. Journal of Vibroengineering, Volume 10, Issue 4, 2008, pp. 510-518.

9. Smirnova R.S., Zakrzhevsky M.V., Schukin I.T., Yevstignejev V.Yu. The Influence of Nonlinear Dissipation on the Birth of Rare Attractors in Nonlinear Dynamics. International Symposium RA11 on "Rare Attractors and Rare Phenomena in Nonlinear Dynamics", Rīga, 2011, pp. 21-24.

10. Smirnova $\mathrm{R}$. The transition to chaos and criteria of chaotic behavior in dynamical systems with linear and nonlinear damping: DYVIS-2012 - the Dynamics of Vibroimpact (Strongly Nonlinear) Systems, Russian Academy of Sciences, Moscow- Klin, 2012, pp. 261-267.

11. Smirnova R., Zakrzhevsky M., Schukin I. Global Analysis of the Nonlinear Duffing-van der Pol Type Equation by a Bifurcation Theory and Complete Bifurcation Groups Method. Vibroengineering Procedia, 2014, Vol.16, Iss.3, 139.-143.lpp. ISSN 2345-0533. 